\title{
Virtual Reality Simulation of Active Car Suspension System
}

\author{
Jason Smoker ${ }^{1}$, Amr Baz ${ }^{1}$ and Ling Zheng ${ }^{2}$ \\ ${ }^{I}$ Department of Mechanical Engineering, University of Maryland, College Park, MD 20742, USA \\ ${ }^{2}$ State Key Laboratory of Mechanical Transmission, Chongqing University, Chongqing 400044, P.R.China
}

\begin{abstract}
This paper presents the design of a Nonlinear Energy Sink (NES) controller and its application to active suspension systems in the Virtual Reality Environment. In this environment, the design engineers are immersed in an audiovisually coupled tele-operated environment whereby direct interaction with and control of the design process is achieved in real time. In this manner, the behavior of synthetic models of the full car can be monitored by literally walking around the car and adjusting the design parameters of the suspension as needed to ensure optimal performance while satisfying design and operational requirements.

The control actuators which provide forces equivalent to nonlinear stiffness and damping elements are attached to the vehicle in order to actively isolate it from road excitation. The effect of the parameters of the NES controller on the vehicle performance is studied both in the frequency and time domain. The effectiveness of the NES controller is validated by numerical simulation. The robustness of the nonlinear energy pumping process is studied by varying the magnitude of road excitation. The simulation results in the Virtual Reality Environment show that under certain conditions, the nonlinear energy pumping can be induced and significant vibration isolation can be achieved. The performance of vehicle including the ride comfort and road holding capability can be improved significantly. When the magnitude of road excitation is increased, the capacity of the NES to absorb energy from the main system is also enhanced. This is very important to achieve vibration isolation objectives. The virtual reality simulation results also show that the nonlinear NES controller performs better than the classical LQR controller particularly as the road condition becomes worst.
\end{abstract}

Index Terms---Design in virtual reality environment, nonlinear energy sinks, LQR, controllable suspension, control strategy.

\section{INTRODUCTION}

In order to improve the vehicle performance, computercontrolled suspensions such as active or semi-active suspension have been considered. Several control strategies have been proposed. Examples of these strategies include: skyhook model-following [1], adaptive [2], linear quadratic Gaussian (LQG) [3], neural network [4], nonlinear $\mathrm{H}_{\infty}[5$ and 6], the mixed $\mathrm{H}_{2} / \mathrm{H}_{\infty}$ [7], sliding mode [8], fuzzy [9], and preview [10] control strategies.

Manuscript received on 30 June 2008

E-Mail: baz@umd.edu
Recently, versions of these strategies have been adopted by many automobile makers which offer controllable suspension systems as an option for their high-end passenger cars. Some examples include the suspensions offered by Mercedes Benz, Toyota, and Jaguar (www.jaguarusa.com/us/en/vehicles). Also, Cadillac Seville and Chevrolet Corvette, are currently employing Delphi's MagneRide semi-active suspension technology (www.delphi.com/pdf/e/magneride.pdf).

In this paper, another control strategy is considered which relies in its operation on Nonlinear Energy Sinks (NES) as means for achieving shock isolation of broadband unwanted disturbances. These NES have been extensively analyzed by Vakakis [11 and 12], Gendelman et al. [13], and Ma et al. [14]. Unlike linear passive sinks, as dynamic absorbers, which can operate only in the neighborhood of a single frequency, and are incapable of effectively attenuating transient disturbances, the NES have been shown to suppress transient disturbances in an irreversible manner [11 and 12].

In this paper, the NES are coupled to the car suspension (primary system) by means of nonlinear springs and dampers. Such nonlinear coupling elements are necessary in order to achieve energy pumping and dissipation of unwanted disturbances in a sufficiently fast timescale. Specifically, the idea of the NESs is used to control the vertical dynamics of a seven degree-of-freedom vehicle in order to achieve a desired vehicle body and wheel performance that ensures a balance between the ride and handling performance.

This paper is organized in six sections. In section 1, a brief introduction is given. Section 2 presents the dynamic equations of a full car model with seven degrees of freedom. Section 3 describes the concept of the NES control design strategy and architecture. A numerical example is given in section 4 and implementation in the virtual reality environment is outlined in Section 5. Section 6 summarizes the main results of this work and gives some recommendation for future applications.

\section{DYANMICS OF A FULL-CAR MODEL}

Assume a vehicle driven on a straight road in a steady-state condition, i.e., with constant thrust and without brake action. In this case, the vertical dynamics of the vehicle including the car body heave, roll, and pitch motions, and the bounce motions of the four wheels. This is a typical seven degrees of freedom characterization of the vertical dynamics used for the computer controlled suspension. The vertical dynamic model is shown in Fig. 1. 
Let $z_{w}=\left[\begin{array}{llll}z_{w 1} & z_{w 2} & z_{w 3} & z_{w 4}\end{array}\right]^{T}$, where $z_{w i}$ is the displacement of the $i^{\text {th }}$ wheel of the vehicle, where $i=1,2$, 3 , and 4 correspond to the front-left, front-right, rear- left and rear-right wheels. Also, the body motion vector $q$ is defined as

$$
q=\left[\begin{array}{lll}
h & r & p
\end{array}\right]^{T}
$$

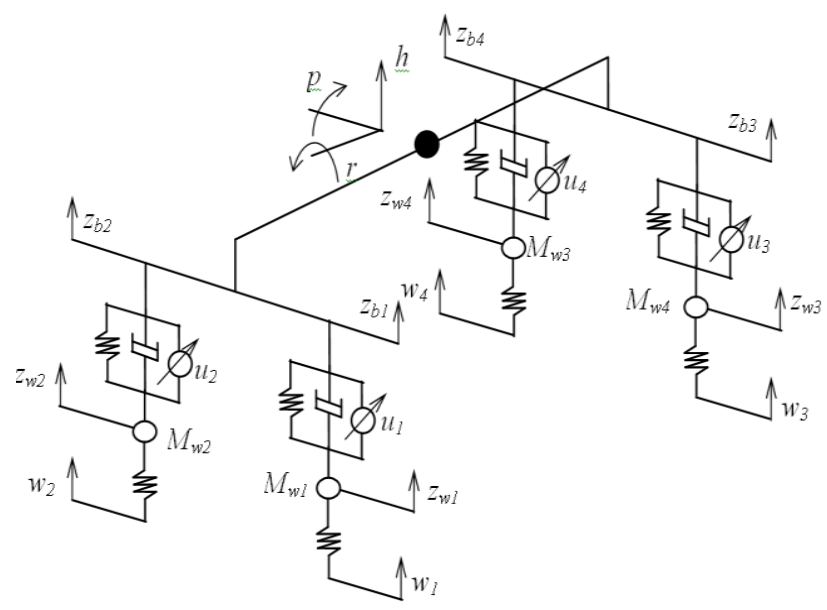

Fig. 1. Seven degrees of freedom full-car model

where $h$ is the heave displacement of the center of gravity of the car body (sprung mass), $\quad r$ is the car body's roll angle, and $p$ is the car body's pitch angle. Both $r$ and $p$ are the global angular displacements with respect to a perfect flat road surface.

\section{Let $\quad Z_{b}=\left[\begin{array}{llll}Z_{1} & Z_{b 2} & Z_{b 3} & Z_{b}\end{array}\right]^{T}$}

, where $z_{b i}$ is the vertical displacement of the car body in the $i^{\text {th }}$ corner, where $i=1, . ., 4$ corresponds to the front-left, front-right, rear- left and rear-right corners. Note that $z_{b}$ and $q$ are related by:

$$
Z_{b}=H q
$$

where $H$ is a transformation matrix given in the appendix. Also, let the relative positions of the suspension be denoted by $z_{r p}$, then:

$$
z_{r p}=z_{b}-z_{w}
$$

Further, let $u$ be the vector of control forces such that:

$$
u=\left[\begin{array}{llll}
u_{1} & u_{2} & u_{3} & u_{4}
\end{array}\right]^{T}
$$

where $u_{i}$ is the control force at the $i^{\text {th }}$ corner.

Hence, the equations of motion of the full-car model are:

$$
M \ddot{\hat{z}}+D \dot{\hat{z}}+K \hat{z}=E_{1} w+E_{2} u
$$

Where $\hat{z}=\left[\begin{array}{ll}q & z_{w}\end{array}\right]^{T}$. Also, the matrices $M, D, K, E_{l}$, and $E_{2}$ are given in the appendix.

In a state-space representation, the equations of motion reduce to:

$$
\dot{\hat{x}}=A \hat{x}+B_{1} w+B_{2} u
$$

where $\hat{x}=\left[\begin{array}{ll}\hat{z} & \dot{z}\end{array}\right]^{T}$. Also, the matrices $A, B_{1}$, and $B_{2}$ are defined in the appendix.

\section{NONLINEAR ENERGY SINK CONTROL}

The NES controller is designed such that the control action $u_{i}$ is given by:

$$
u_{i}=-N_{i} g_{i}
$$

where $g_{i}=\left[\begin{array}{llll}\left(z_{b i}-z_{w i}\right)^{3} & \dot{z}_{b i} & z_{w i}-w_{i} & \dot{z}_{w i}{ }^{3}\end{array}\right]^{T}$ and $N_{i}$ is the feedback gain matrix of the $i^{\text {th }}$ NES controller which is given by:

$$
N_{i}=\left[\alpha_{1 i}, \alpha_{2 i}, \alpha_{3 i}, \alpha_{4 i}\right]
$$

It can be seen that nonlinear stiffness element $\left(z_{b i}-z_{w i}\right)^{3}$ and nonlinear damping element $\dot{z}_{w i}{ }^{3}$ have been introduced into the subsystems of the vehicle.

Note that in the proposed control strategy, four independent NES controllers have been used for each quarter car. The parameters $\alpha_{i}$ for the gain matrix are selected to optimize the system performance.

\section{PERFORMANCE OF THE NES CONTROLLER}

The performance of the NES controller is evaluated for a vehicle with the parameters listed in Table 1 . The optimal control gain matrices of the NES are:

$$
\begin{aligned}
N_{1} & =N_{2}=[20000,2203.5,205.2,-1500] \\
\text { and } \quad N_{3} & =N_{4}=[20000,2803.5,805.2,-1500]
\end{aligned}
$$

TABLE 1: VEHICLE PARAMETERS

\begin{tabular}{lcc}
\hline \multicolumn{1}{c}{ Parameter } & Unit & Value \\
\hline$M_{s}$ & $k g$ & 1583 \\
$I_{x x} / I_{y y}$ & $k g m^{2}$ & $531 / 2555$ \\
$M_{w 1} / M_{w 2} / M_{w 3} / M_{w 4}$ & $k g$ & $48 / 48 / 74 / 74$ \\
$K_{s 1} / K_{s 2} / K_{s 3} / K_{s 4}$ & $K N / m$ & $35 / 35 / 34 / 34$ \\
$C_{s 1} / C_{s 2} / C_{s 3} / C_{s 4}$ & $N s / m$ & $400 / 400 / 200 / 200$ \\
$K_{t 1} / K_{t 2} / K_{t 3} / K_{t 4}$ & $K N / m$ & $220 / 220 / 220 / 220$ \\
$I_{x f} / I_{x r}$ & $m$ & $1.116 / 1.438$ \\
\hline$l_{y l f} / l_{y r f} / l_{y l r} / l_{y r r}$ & $m$ & $0.77 / 0.77 / 0.765 / 0.765$ \\
\hline
\end{tabular}

In this paper, chirp signal road profiles are considered. Typical profiles are shown in Fig. 2, indicating dominant 
frequency content from 0 to $15 \mathrm{~Hz}$ [7]. Also, the signals for the left and right wheels are assumed to have a frequency shift in order to excite the pitch and roll modes of the car body. In addition, the vehicle is assumed to be driven straight at a constant speed of $60 \mathrm{~km} / \mathrm{h}$.

Fig. 3 shows comparisons between the performance of a passive suspension system and an active system controlled by an LQR controller.

The gain matrices of the LQR controller are:

and

$$
k_{1}=k_{2}=[-1762.6,846,789.6,3.3]
$$

$$
k_{3}=k_{4}=[-2716.8,1078.2,1486.1,-58.9]
$$

Fig. 4 and 5 show comparisons between the performance of the NES and LQR controllers both in the time and frequency domains.It can be clearly seen that the NES control strategy is more effective in attenuating the vibration of the vehicle body and improving the handling performance. Furthermore, the suspsion deflections are also attenuated at the same time
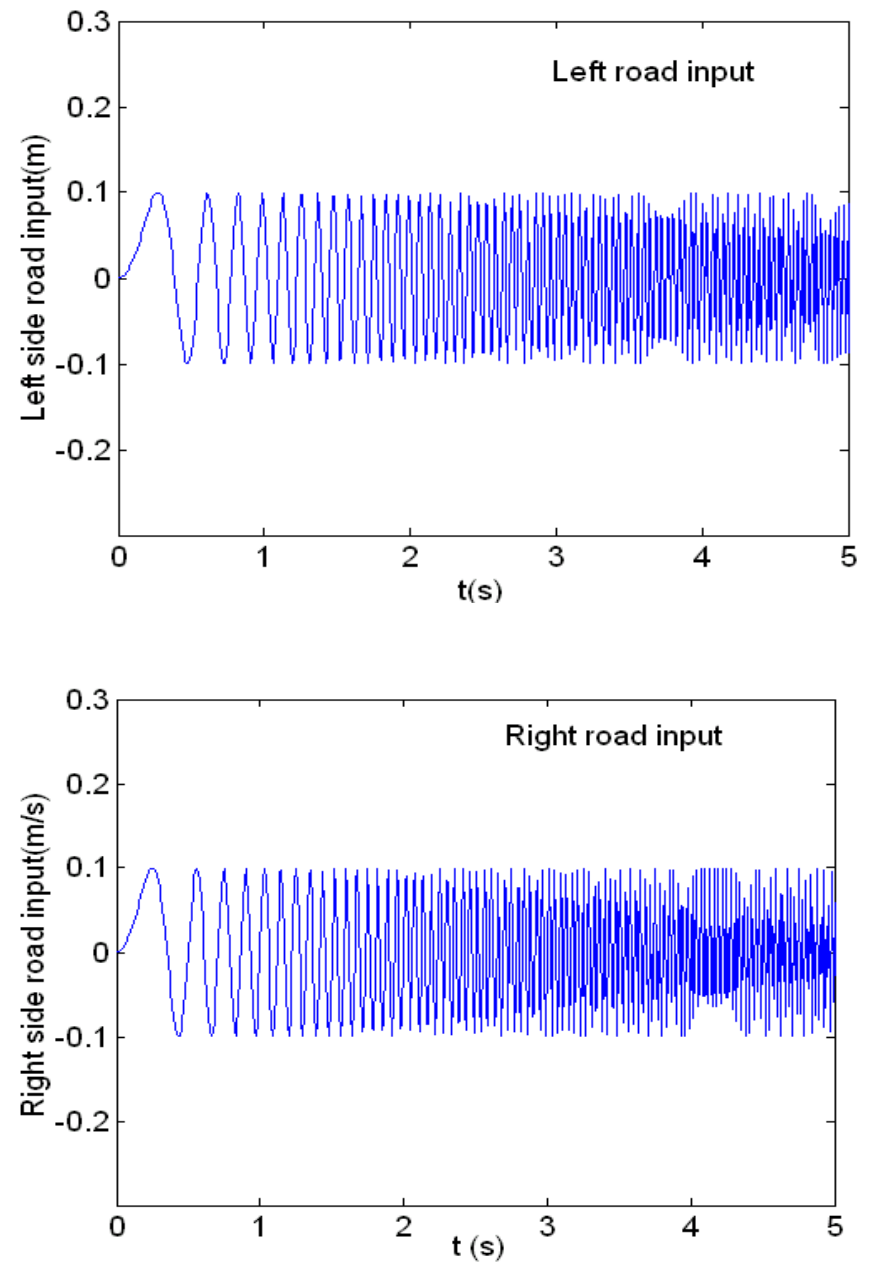

(a) time domain

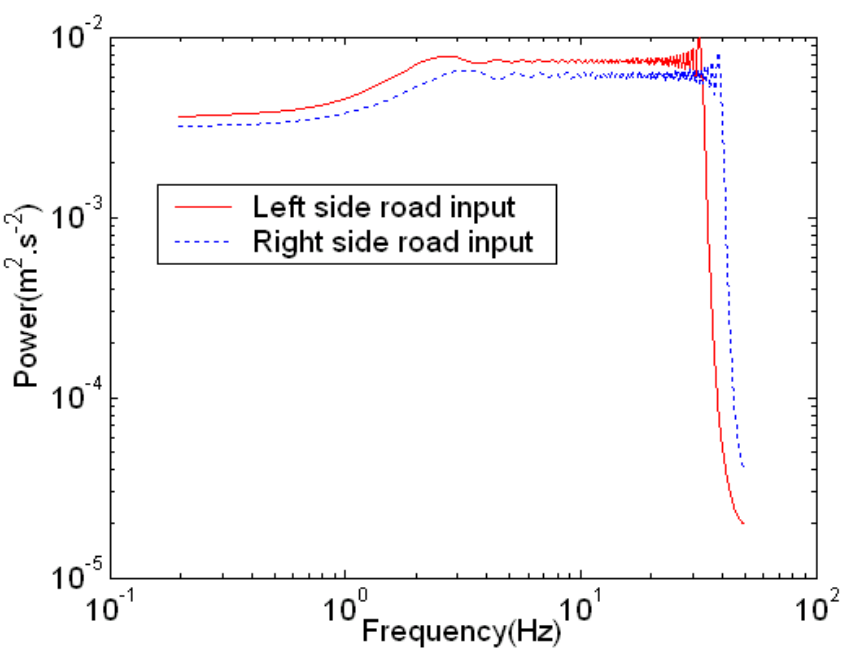

(b) frequency domain

Fig. 2. Time profile and frequency content of chirp road inputs

\section{IMPLEMENTATION IN THE VIRTUAL REALITY EN- VIRONMENT}

The dynamics of the full-car model with the NES controller are implemented in the University of Maryland Virtual Reality (CAVE) Laboratory which is controlled by an eight parallel processor Silicon Graphics Infinite Reality (ONYX2) computer as shown in Fig. 6. In the virtual reality environment, the designer becomes an integral part of the computer-aided design process and the design loop. This is very important as the vehicle designer by being immersed in the virtual reality environment can view the attributes of each of the control strategies in real time and gain considerable insight about the physics of the system. He can favor one controller design over another or modify the design parameters to capture the physics of the problem. Other examples of virtual reality simulation of vehicle dynamics are reported by Ozana [6], Lehner and DeFanti [15], Belyaev and Aranov [16], and Kumar et al. [17].

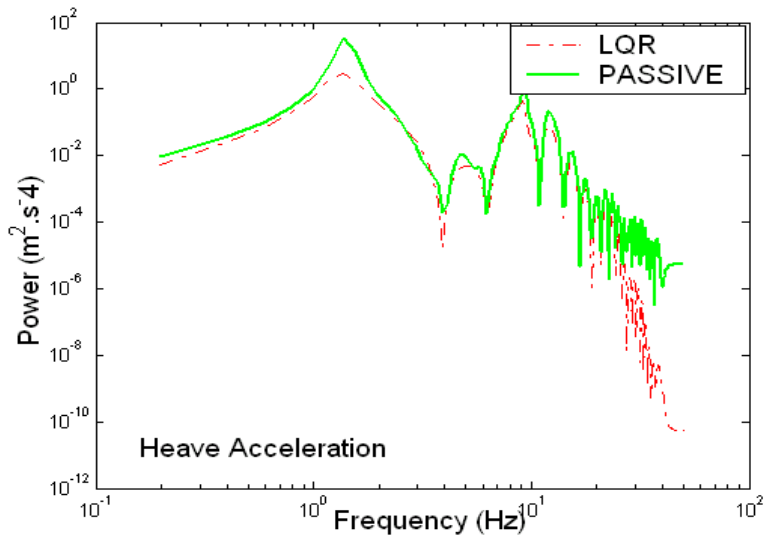



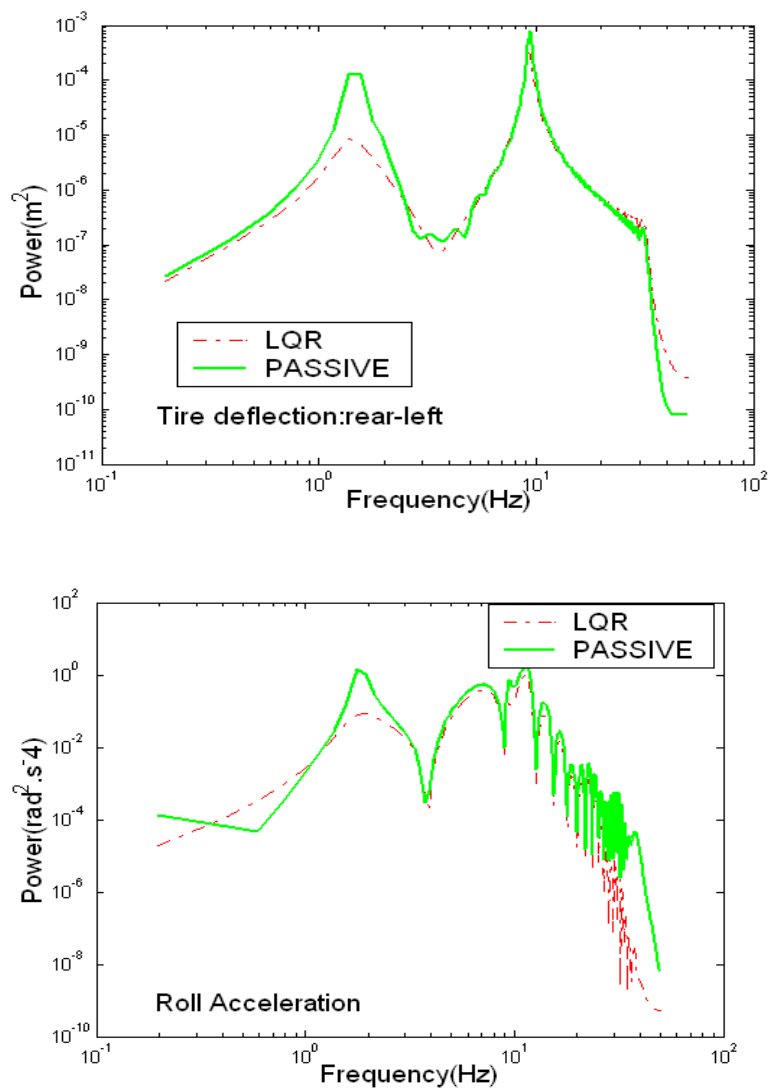

Fig. 3. Frequency response of vehicle with $L Q R$

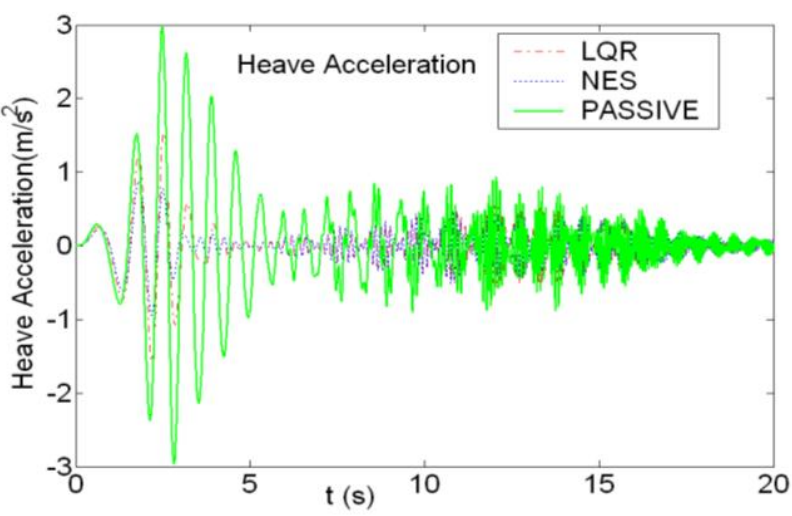

(a)

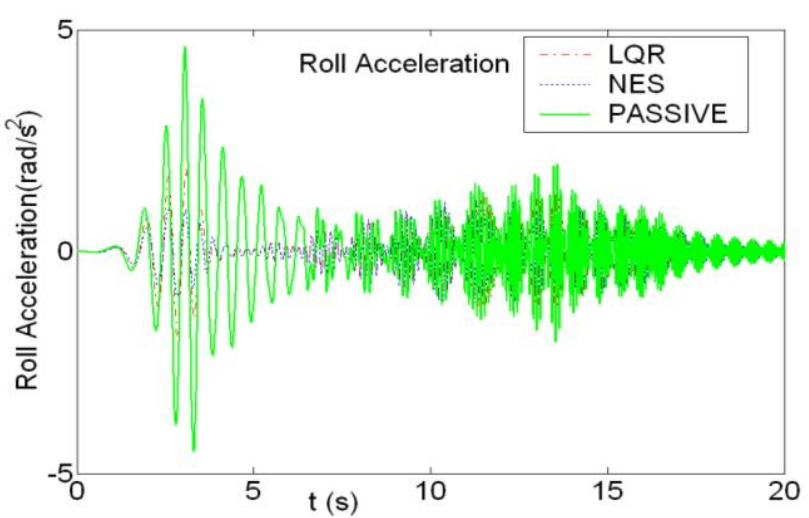

(b)

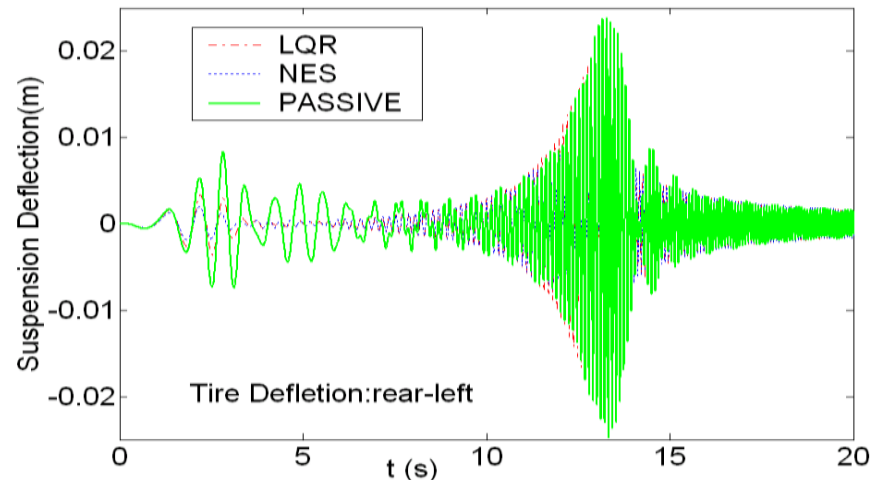

(c)

Fig. 4. Time domain
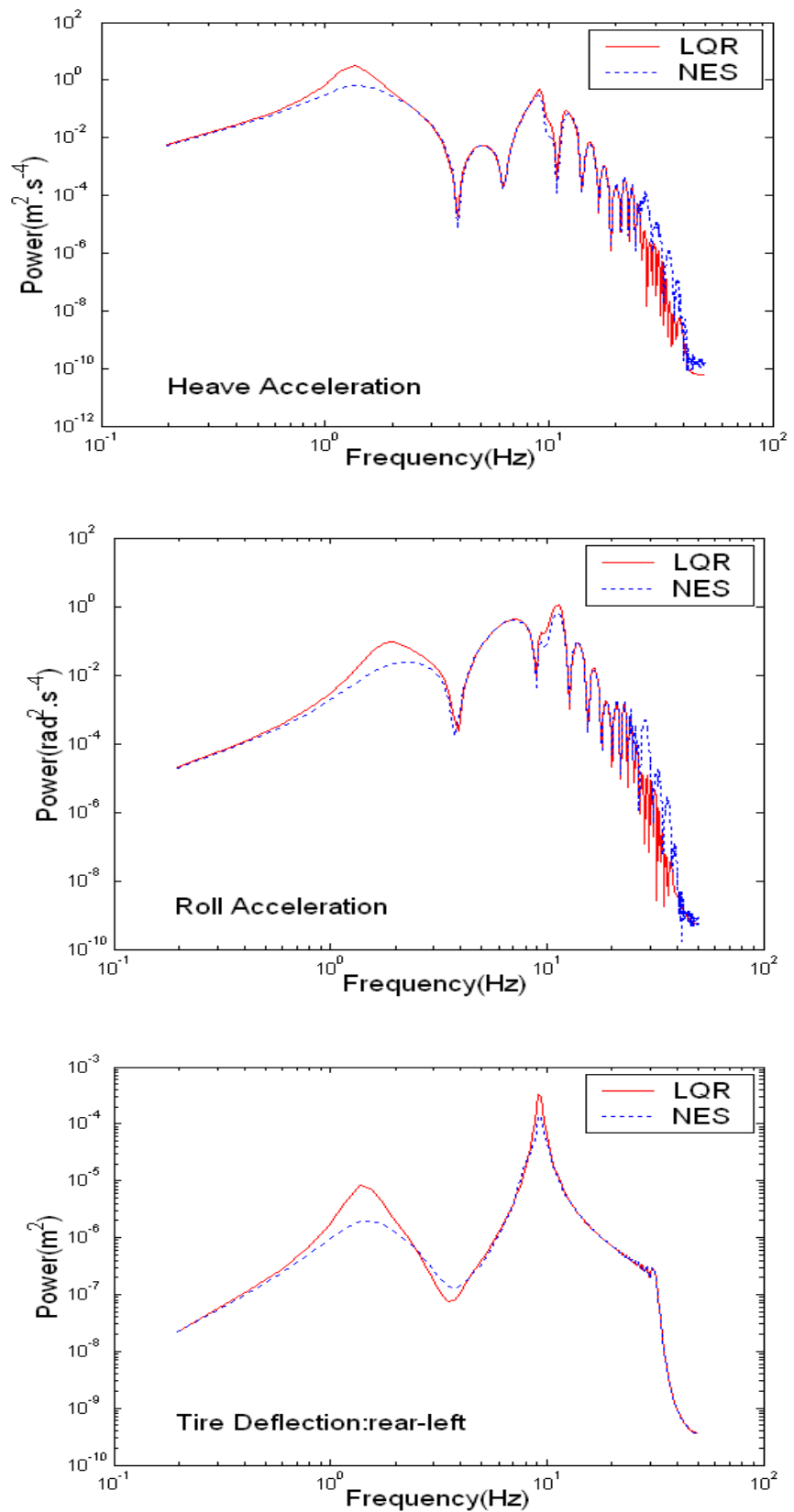

Fig. 5. Frequency domain 


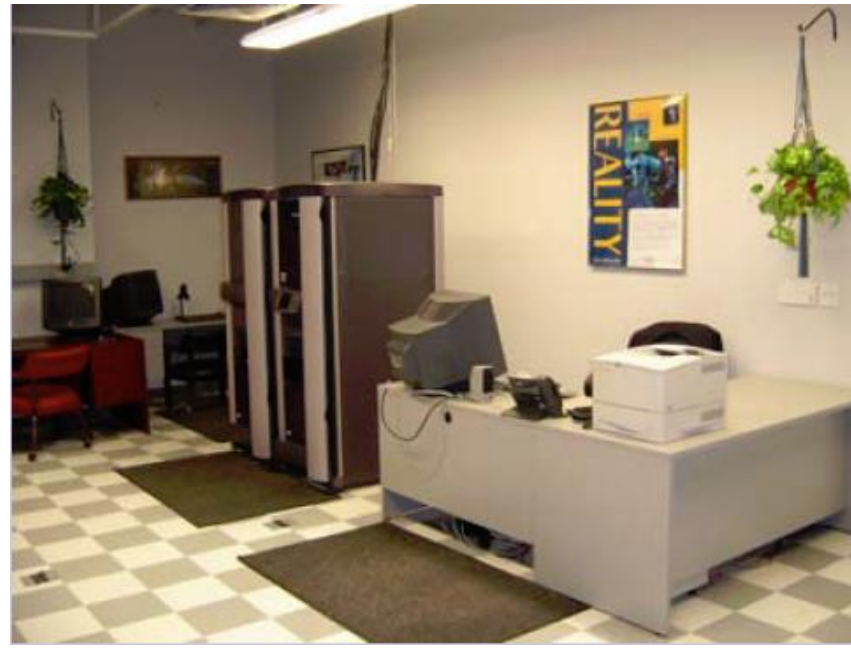

(a) SGI-ONYX Computer

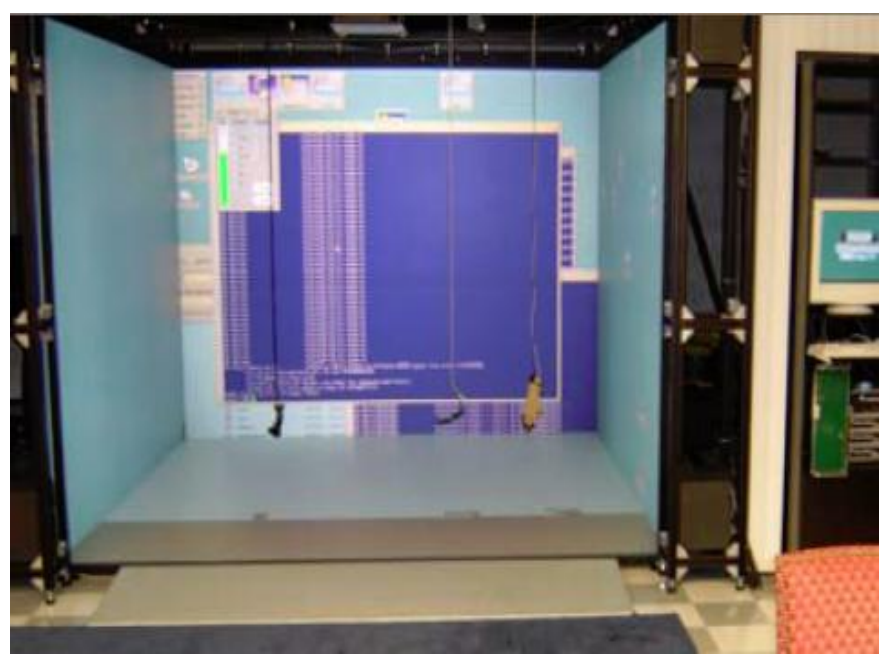

(b) CAVE

Fig. 6. Virtual reality laboratory at University of Maryland

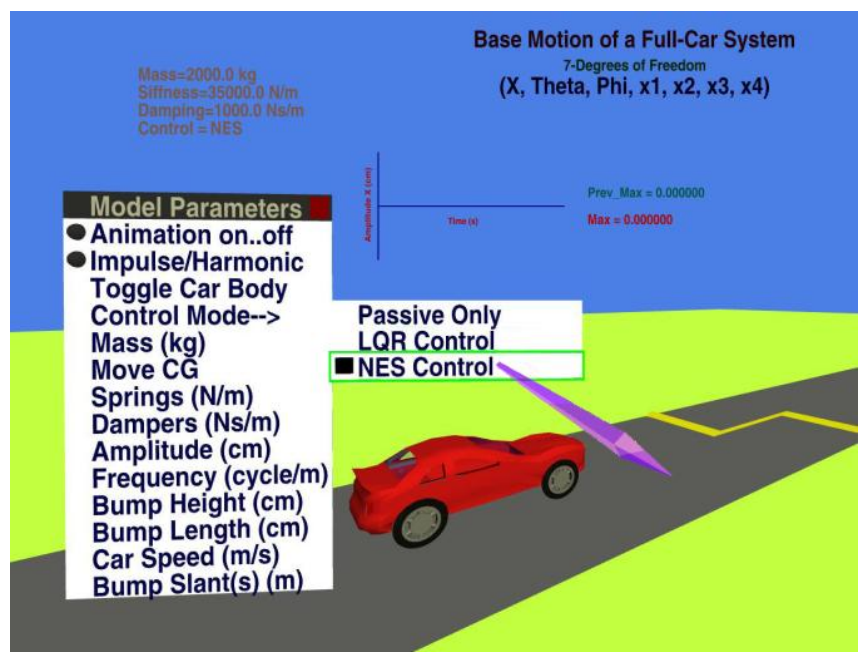

Fig. 7. The main menu of analysis and design of vehicle suspension in virtual reality environment

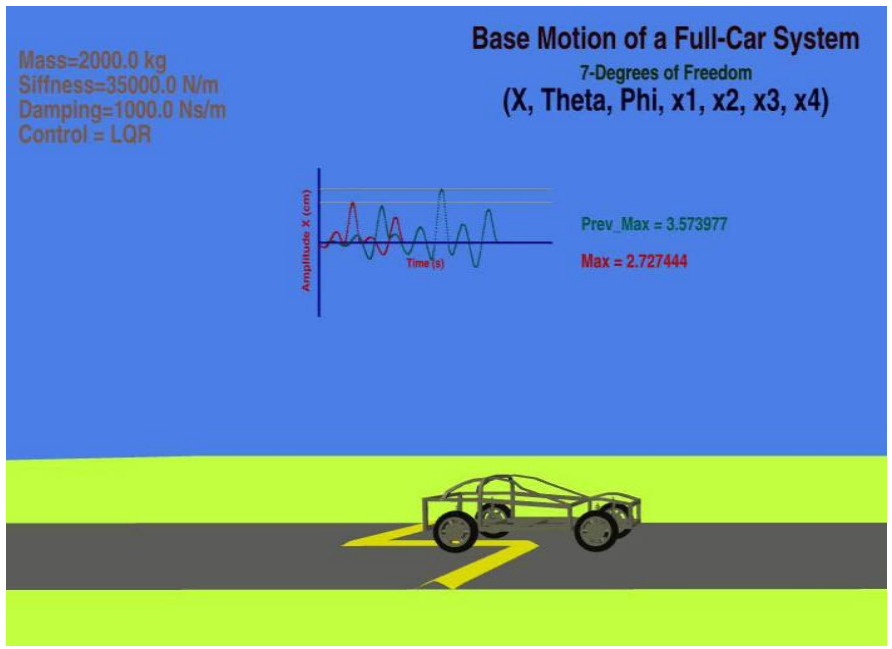

Fig. 8. Display of the design parameters and the corresponding performance characteristics

Fig. 7 shows the main menu of the analysis and design of active vehicle suspension systems in the virtual reality environment. In such an environment, the designer can investigate the effect of the car suspension parameters, the road excitation, car speed, and control strategy on the car dynamics in real time.

Fig. 8 shows, in real time, a comparison between the time response of a vehicle with passive suspension (in green) and a vehicle with an NES controller (in red) when the car passes over a speed bump. Attenuation of the peak amplitude of vibration is evident.

Fig.s 9 through 11 display comparisons between the dynamics of a passive (uncontrolled) vehicle and vehicles controlled with the LQR and NES controllers for heave, pitch, and roll degrees of freedom.

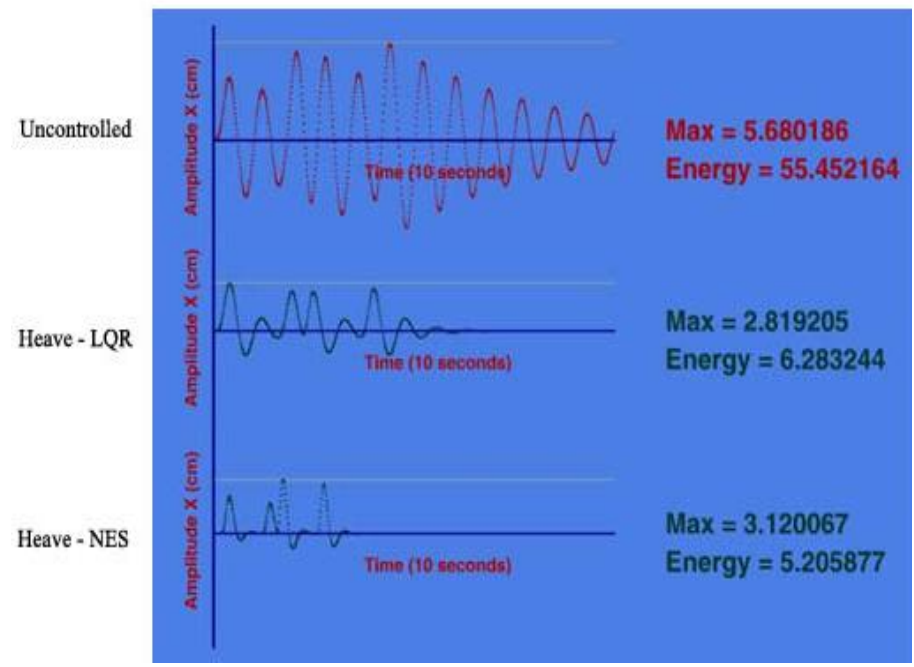

Fig. 9. Comparison of heave dynamics 


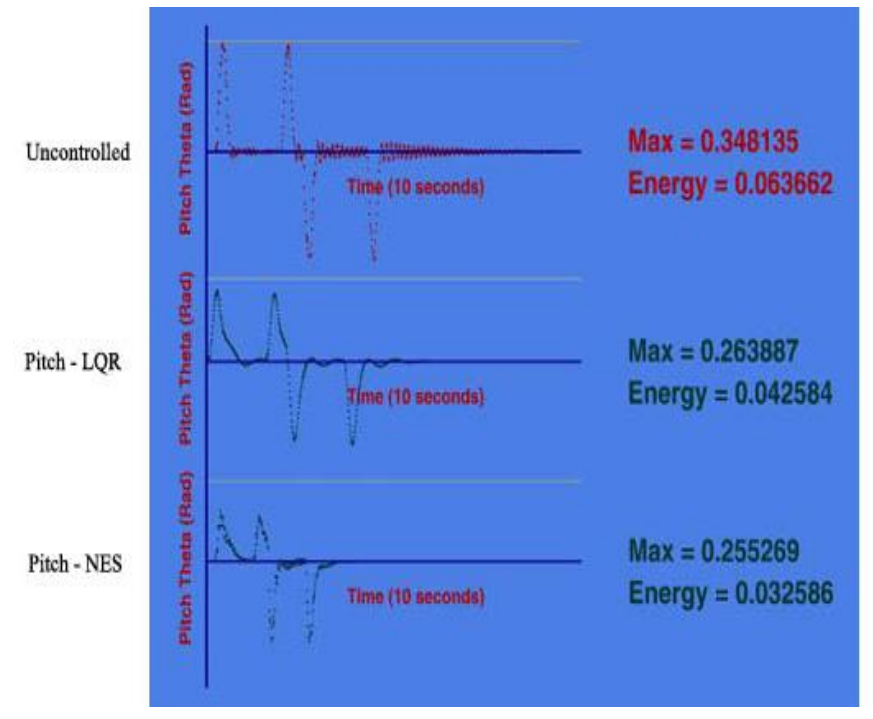

Fig. 10. Comparison of pitch dynamics

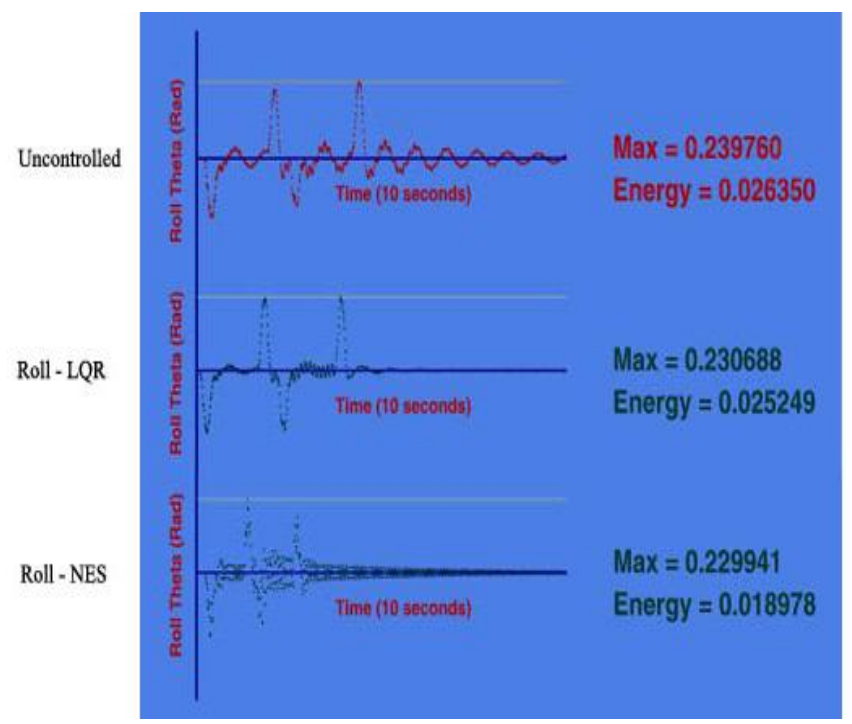

Fig. 11. Comparison of roll dynamics

\section{CONCLUSIONS}

This paper has presented the implementation of the dynamics of a seven-degree of freedom vehicle with a nonlinear energy sink controller in the virtual reality environment. The implementation aims at demonstrating the effectiveness of a nonlinear energy sink (NES) controller as compared to the classical LQR controller and uncontrolled suspension.

The simulation results show that NES controllers are more effective than LQR controllers and passive system whether for ride comfort (body performance) or handling performance (wheel performance). With proper selection of the control gains, the NES can generate effective nonlinear energy pumping over a wide frequency range whereby the vibration energy produced by the road disturbance are absorbed into nonlinear attachments and thus an effective vibration isolation is obtained for vehicle.
It is important to note that the design of car suspension systems in the virtual environment enables the design engineer to be fully immersed in an audio-visually coupled tele-operated environment where the physical performance of the suspension can be monitored by literally walking around the car and adjusting its design parameters as needed to ensure optimal performance while satisfying design and operational requirements. Under such conditions, the With such an addition, the designer becomes an integral part of the computer aided design (CAD) process and the design loop where he can make decisions based on the insight gained during the direct interaction with virtual model of the car. Further benefits can be gained when the virtual reality environment is used in the collaborative design mode where several engineers at different sites can share their inputs without physically be at one place.

However, one must emphasize that extensions of the present work are now in progress in order to enhance its practicality and potential. For example, a coupled control system is being developed to improve the performance as compared to the independent wheel controllers considered in this paper. Furthermore, the dynamics of the actuators and the sensors are in the process of being accounted for to ensure practicality of the suspension system. Validation of the optimized configuration experimentally is a natural extension of the present work.

The presented work can also be integrated with driving simulators such as that developed by Sun et al. [18] and cognitive response capabilities as reported by Lin et al. [19] in order to establish comprehensive virtual reality facilities for car/driver systems. Also, the presented large scale virtual reality simulation in CAVE environment can be equally extended to other vehicle systems such as bicycles [20] and Unmanned Air Vehicle (UAV) [21].

\section{APPENDIX}

\section{MATRICES OF CAR MODEL}

\section{i. Transformation Matrix}

$$
H=\left[\begin{array}{ccc}
1 & l_{y l f} & -l_{x f} \\
1 & -l_{y l f} & -l_{x f} \\
1 & l_{y l r} & l_{x r} \\
1 & -l_{y l r} & l_{x r}
\end{array}\right]
$$

where $l_{x f}$ and $l_{x r}$ are the distances from the front and rear axle to car body CG, respectively, $l_{y l f}$ and $l_{y l r}$ are half of the front and rear wheel tracks.

\section{ii. Mass, Stiffness, and Damping Matrices}




$$
\begin{aligned}
M_{b} & =\left[\begin{array}{ccc}
M_{S} & 0 & 0 \\
0 & I_{x x} & 0 \\
0 & 0 & I_{y y}
\end{array}\right], M_{w}=\left[\begin{array}{cccc}
M_{w 1} & 0 & 0 & 0 \\
0 & M_{w 2} & 0 & 0 \\
0 & 0 & M_{w 3} & 0 \\
0 & 0 & 0 & M_{w 4}
\end{array}\right] \\
K_{S} & =\left[\begin{array}{cccc}
K_{S 1} & 0 & 0 & 0 \\
0 & K_{S 2} & 0 & 0 \\
0 & 0 & K_{S 3} & 0 \\
0 & 0 & 0 & K_{S 4}
\end{array}\right], C_{S}=\left[\begin{array}{cccc}
C_{S 1} & 0 & 0 & 0 \\
0 & C_{S 2} & 0 & 0 \\
0 & 0 & C_{S 3} & 0 \\
0 & 0 & 0 & C_{S 4}
\end{array}\right]
\end{aligned}
$$

and

$$
K_{t}=\left[\begin{array}{cccc}
K_{t 1} & 0 & 0 & 0 \\
0 & K_{t 2} & 0 & 0 \\
0 & 0 & K_{t 3} & 0 \\
0 & 0 & 0 & K_{t 4}
\end{array}\right]
$$

where $M_{s}$ is sprung mass, $I_{x x}$ and $I_{y y}$ are the roll and pitch moments of inertia of the car body. $M_{w i}$ is the unsprung mass at the $i^{\text {th }}$ corner, $K_{t i}$ is the $i^{\text {th }}$ tire stiffness. $K_{s i}$ as the passive suspension spring rate at the $i^{\text {th }}$ corner, and $C_{s i}$ as the passive damping rate at the $i^{\text {th }}$ corner.

$$
\begin{aligned}
& M=\left[\begin{array}{cc}
M_{b} & 0 \\
0 & M_{w}
\end{array}\right], K=\left[\begin{array}{cc}
H^{\prime} K_{S} H & -H^{\prime} K_{S} \\
-K_{S} H & K_{t}+K_{S}
\end{array}\right] \\
& \text { and } \quad D=\left[\begin{array}{cc}
H^{\prime} C_{S} H & -H^{\prime} C_{S} \\
-C_{S} H & C_{S}
\end{array}\right] \\
& \text { Also, } \quad E 1=\left[\begin{array}{c}
0 \\
K_{t}
\end{array}\right] \quad \text { and } \quad E 2=\left[\begin{array}{c}
H \\
-I
\end{array}\right]
\end{aligned}
$$

\section{iii. Matrices of state-space model}

$$
A=\left[\begin{array}{cc}
0 & I \\
-M^{-1} K & -M^{-1} D
\end{array}\right], B_{1}=\left[\begin{array}{c}
0 \\
M^{-1} E_{1}
\end{array}\right]
$$

and

$$
B_{2}=\left[\begin{array}{c}
0 \\
M^{-1} E_{2}
\end{array}\right]
$$

\section{REFERENCES}

[1] H. Yoshida. and M., Nagai, 1998. Sky-hook model following control of vehicle suspension using an electoro-rheological damper, Transaction of Japan society of Mechanical Engineer, 64625C:3420-3426.

[2] I. Youn. and A., Hac, 1995. Semi-active suspensions with adaptive capability, Journal of Sound and Vibration, 180 (3): 475-492.

[3] L. Zuo. and S.A., Nayfeh, 2003. Structured H2 optimization of vehicle suspensions based on multi-wheel models, Vehicle System Dynamics, 40 (5): 351-371.
[4] W., Kozukue. and H., Miyaji, 2004. Control of vehicle suspension using neural network, Vehicle System Dynamics Supplement, 41:153161

[5] S. Ohsaku, T., Nakayama, I., Kamimura. and Y., Motozono, 1999. Nonlinear $\mathrm{H} \infty$ control for semi-active suspension, JSAE Review, 20:447-452.

[6] S. Ožana, 2008, Visualization of Active Suspension by Robust Controller in Virtual Reality Toolbox, Proceedings of the 2008 19th International Conference on Systems Engineering, IEEE Computer Society, 10.1109/ICSEng.2008.83, 38-42.

[7] J.B. Lu, and D.P. Mark, 2002. Multiobjective optimal suspension control to achieve integrated ride and handling performance, IEEE Trans. Control Systems Technology, 10(6): 807-821.

[8] N. Yagiz, and I. Yuksek, 2001. Sliding mode control of active suspension for a full vehicle model, International Journal of Vehicle Design, 26(2/3):264-265.F.J. Damato. and D.E. Viassolo, 2000. Fuzzy control for active suspensions, Mechatronics, 10:897-920.

[9] T.J. Gordon. and R.S. Sharp, 1998. On improving the performance of automotive semi-active suspension systems through road preview, Journal of Sound and Vibration, 217 (1):163-182.

[10] A.F. Vakakis, 2001. Inducing passive nonlinear energy sinks in vibrating systems, Journal of Vibration and Acoustics, 123(3):324-332.

[11] A. F. Vakakis, 2003. Shock isolation through the use of nonlinear energy sinks, Journal of Vibration and Control, 9:79-93.

[12] O. Gendelman, L. I. Manevitch, A. F. Vakakis, and R. M'Closkey, 2001. "Energy pumping in nonlinear mechanical oscillators: Part Idynamics of the underlying hamiltonian systems", ASME Journal of Applied Mechanics, 68: 34-41.

[13] X. Ma, T. A. Nayfeh, A. F. Vakakis and L. A. Bergman, 2000. Experimental verification of shock reduction achieved through non-linear localization, Journal of Sound and Vibration, 230 (5): 1177-1184.

[14] V. Lehner and T. DeFanti, 1997. Distributed virtual reality: supporting remote collaboration in vehicle design," IEEE Comp. Graphics and Appl., 17 (2): 13-17.

[15] S.Y. Belyaev and V.Y. Aranov, 2001. Numerical simulation of vehicle dynamics for virtual reality, Fourth International Workshop on Nondestructive Testing and Computer Simulations in Science and Engineering, edited by A. I. Melker, 4348:405-412.

[16] M.M. Kumar, T.Shim and J. Asgari, 2007, Development of a vehicle animation tool using MATLAB virtual reality toolbox, SAE World Congress \& Exhibition, April 2007, Detroit, MI, 2007-01-0947.

[17] C.Sun, F. Xie, Feng X., M. Zhang, and Z. Pan, 2007. A Training Oriented Driving Simulator", Proceeding of 6th International Conference on Entertainment Computing (ICEC 2007), edited by L. Ma, M. Rauterberg, and R. Nakatsu, Shanghai, China, September 15-17, 2007, Vol. 4740 Springer 2007, 1-9.

[18] C. Lin., L. Chung., L. Ko., Y. Chen., S. Liang, J. Duann, 2007, EEG-Based Assessment of Driver Cognitive Responses in a Dynamic Virtual-Reality Driving Environment, IEEE Transactions on Biomedical Engineering, 54 (7): 1349-1352.

[19] O. Schulzyk, U. Hartmann, J. Bongartz, T. Bildhauer, and R. Herpers, 2009. A real bicycle simulator in a virtual reality environment: the FIVIS project", Proceedings of 4th European Conference of the International Federation for Medical and Biological Engineering (IFMBE), $22: 2628-2631$

[20] Z. Qiao, Y. Li, S. Kang and Q. Zhu, 2008, Design of UAV Telepresence and Simulation Platform Based on VR. IEEE International Conference on Cyberworlds, 520-524.

\section{SYMBOLS}

$$
\begin{array}{cl}
C_{s i} & \text { passive damping rate at the } i^{\text {th }} \text { corner } \\
H & \text { transformation matrix } \\
h & \text { heave displacement of CG of car body (sprung mass) } \\
I_{x x}, I_{y y} & \text { roll and pitch moments of inertia of the car body } \\
K_{t i} & \text { Stiffness of the } i^{\text {th }} \text { tire } \\
K_{s i} & \text { passive suspension spring rate at the } i^{\text {th }} \text { corner }
\end{array}
$$



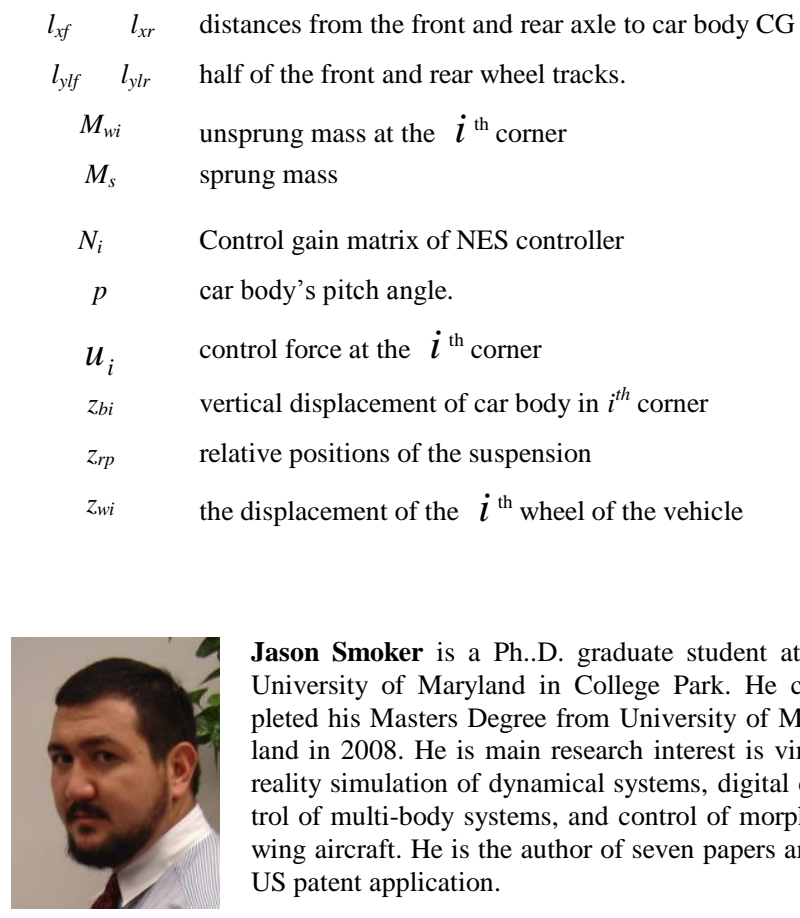

Jason Smoker is a $\mathrm{Ph} . . \mathrm{D}$. graduate student at the University of Maryland in College Park. He completed his Masters Degree from University of Maryland in 2008 . He is main research interest is virtual reality simulation of dynamical systems, digital control of multi-body systems, and control of morphing wing aircraft. He is the author of seven papers and 1 US patent application.

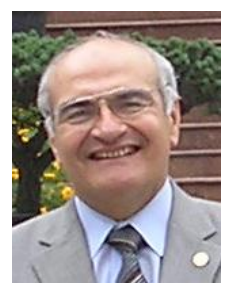

Amr Baz earned his Ph.D. and M.Sc. degrees in Mechanical Engineering from the University of Wisconsin, Madison in 1973 and 1970. He is Full Professor of Mechanical Engineering, and Director of the Smart Materials and Structures Research Center at the University of Maryland in College Park. He is the author of more than 150 journal papers and six US patents. In 2009, he received the ASME Adaptive Structures \& Materials Award for significant contributions to the science and technology of adaptive structures.

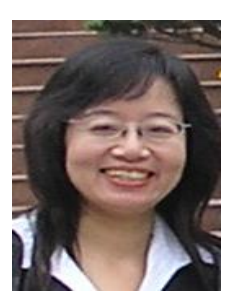

Ling Zheng completed her Ph.D. in Mechanical Engineering at Chongqong University (Chongqing, China). She worked as a member of the Automobile Engineering faculty at the Chongqing Institute of Communications between 1984-1999. Since 1999, she has been a member of the Automobile Engineering faculty at Chongqong University (Chongqing, China) as Professor. Her main research interests are vehicle NVH, active or semi-active sound/vibration control, vehicle system dynamics and structure optimum design. She holds three patents on ER (Electrorheological) fluid, ER damper, MR (Magnetorheological) mount and has published over fifty papers in international journals and conferences. She is also the member of Chinese Society of Mechanical Engineering (CSME). 